\title{
Fibroma desmoplásico de maxilar en paciente pediátrico: Reporte de caso
}

Roberto Gerber Mora ${ }^{1}$

Sergio Castro Mora $^{2}$

Joseph Ulate Jiménez $z^{3}$

\section{Resumen}

La presencia de tumefacciones en la cavidad bucal del paciente pediátrico puede ser indicativo de gran cantidad de patologías y enfermedades sistémicas, algunas incluso pueden perjudicar el bienestar del mismo. El Fibroma desmoplásico es un tumor óseo benigno, localmente destructivo, catalogado como muy poco frecuente. Se reporta el caso de un niño de 4 años con fibroma desmoplásico en el maxilar de alrededor de 2 años de evolución, observándose como una tumefacción generalizada del segundo cuadrante, con ausencia de signos y síntomas de inflamación. Por su edad representa un reto para el clínico ya que el abordaje debe ser interdisciplinario con profesionales expertos en odontopediatría, cirugía y patología. Se describe a profundidad cada paso del proceso diagnóstico incluyendo hallazgos clínicos, radiográficos e histológicos, así como la toma de la muestra para su estudio.

Palabras clave: Fibroma desmoplásico, odontopediatría, maxilar.

Relato de caso

\section{Fibroma desmoplásico em maxila de paciente pediátrico: Relato de caso}

\section{Resumo}

A presença de tumefações na cavidade oral de pacientes pediátricos podem ser indicativa de muitas patologias e enfermidades sistêmicas. Algumas, inclusive, podem prejudicar o bemestar da criança. O fibroma desmoplásico é um tumor ósseo benigno localmente destrutivo, listado como raro. Nesse artigo, um caso de uma criança de 4 anos com fibroma desmoplásico no maxilar com cerca de dois anos de evolução é relatado. Observava-se uma tumefação generalizada do segundo quadrante, com ausência de sinais e sintomas

\footnotetext{
${ }^{1}$ Odontólogo General, Pasantía en Cirugía y Patología Oral, Universidad Latina de Costa Rica.

${ }^{2}$ Especialista en Patología oral, Medicina Oral y Maxilofacial, Docente Universidad de Costa Rica. Docente Universidad Latina de Costa Rica.

${ }^{3}$ Odontólogo Pediatra, Docente Universidad de Costa Rica. Docente Universidad Latina de Costa Rica
} 
de inflamação. Devido à idade, representa um desafio para o clínico, já que a abordagem deve ser interdisciplinar com especialistas em odontopediatria, cirurgia, patologia. Se descreve aprofundadamente cada etapa do processo de diagnóstico, incluindo achados clínicos, radiográficos e histológico.

Palavras-chave: fibroma desmoplásico odontopediatria, maxila.

\section{Critical Review Article}

\section{Desmoplastic fibroma of the maxillae in a pediatric patient: Case report}

\begin{abstract}
The presence of swellings in the oral cavity in pediatric patients may be indicative of a large number of pathologies and systemic diseases; some may even impair the childs wellbeing. The desmoplastic fibroma is a locally destructive benign bone tumor, listed as rare. A case of a 4-year old boy with a two year developing desmoplastic fibroma in the maxillae is reported. Because of his age, it represents a challenge for clinicians, and the approach should be interdisciplinary, involving experts in pediatric dentistry, oral surgery and oral pathology. Every step of the diagnostic process is described in depth, including clinical, radiographic and histological findings.
\end{abstract}

Key Words: Desmoplastic fibroma, pediatric dentistry, maxilla.

\section{Introducción}

Reportado por primera vez en 1958 por Jaffe $^{1}$, el fibroma desmoplásico (FD) es un tumor intraóseo sumamente infrecuente ${ }^{2}, y$ aunque es de naturaleza benigna, presenta un comportamiento localmente destructivo ${ }^{3}$. Las zonas más comunes de desarrollo comprenden los huesos largos ${ }^{4}$, la mandíbula es considerada el cuarto sitio de predilección. Con menos de 200 publicaciones conocidas sobre esta entidad ${ }^{5}$, el FD representa tan sólo el $0.06 \%$ de todos los tumores de hueso, y el $0.3 \%$ del total de tumores benignos óseos ${ }^{6}$. Aunque su etiología es desconocida, algunos autores refieren alteraciones endocrinas, genéticas o traumáticas como factores en la patogenia de la lesión y que podrían hacer pensar en una lesión reactiva7.

Los signos y síntomas clínicos que puede presentar un paciente con FD no son patognomónicos de la misma ${ }^{8}$, y aunque en la mayoría de casos se refiere una evolución asintomática, algunos reportes describen dolor y/o pérdida de la función de la zona afectada. La edad media de diagnóstico ronda los 15 años ${ }^{9}$, aun así se han documentado casos en pacientes desde los 12 meses hasta la séptima década de vida7. A pesar de que las regiones metafisiarias del humero y radio cuentan como las zonas más frecuentes para el desarrollo de este tipo de anomalías, el hueso mandibular es considerado la cuar- 


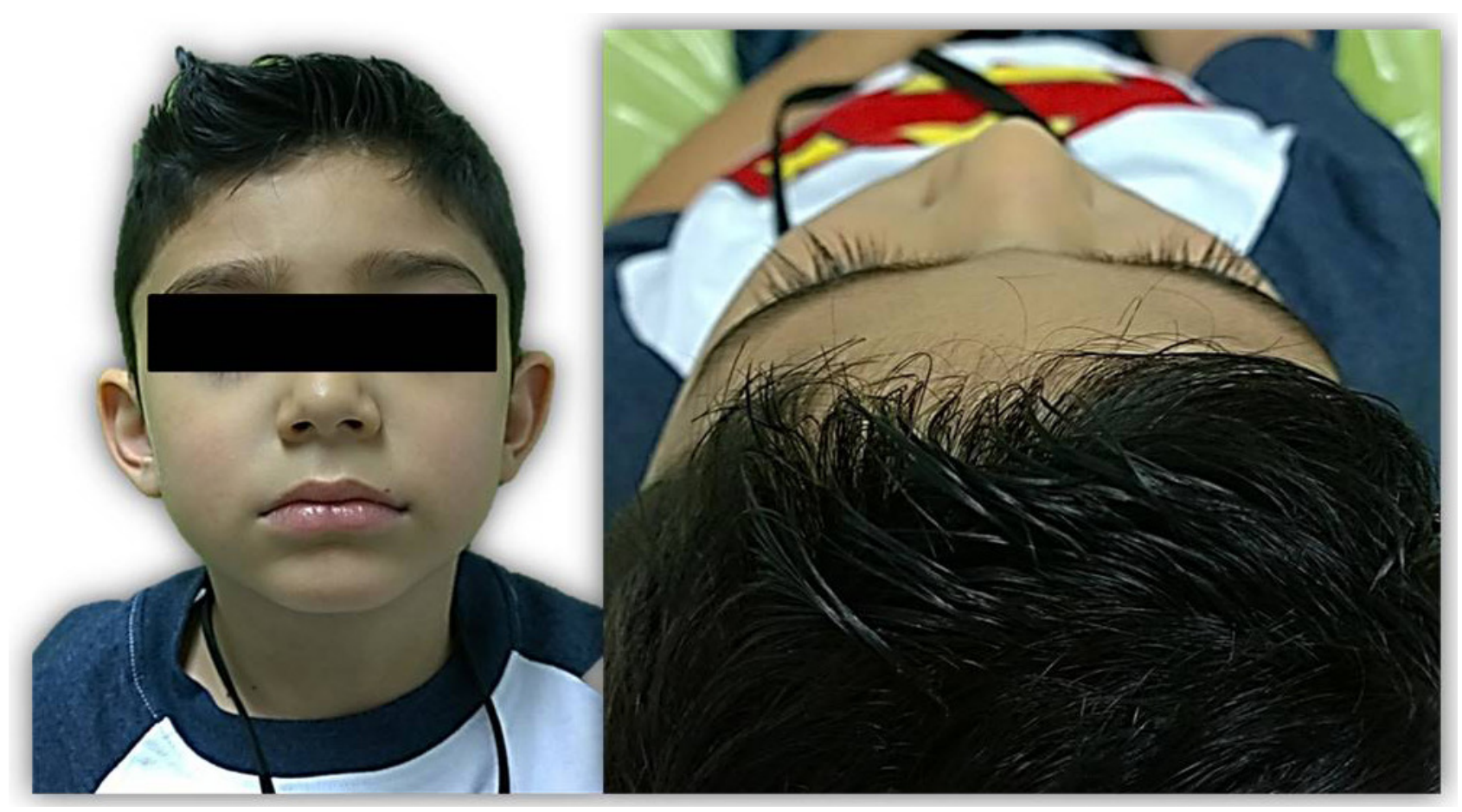

Figura 1 y 2.Paciente masculino de 4 años de edad con un leve abultamiento asintomático a nivel de la mejilla izquierda

ta locación en ser afectada por el FD ${ }^{10}$. Radiográficamente se dificulta distinguirla de otras lesiones óseas ${ }^{11}$, esto a razón de que el FD puede generar diferentes presentaciones tanto radiológicas como clínicas ${ }^{12}$. Un FD puede observarse radiográficamente como una lesión radiolucida con márgenes bien demarcados o pobremente delimitados, puede ser unilocular o multilocular ${ }^{10}$. Aunque no produce metástasis, si presenta una alta tasa de recidiva $^{13}$, no obstante este porcentaje es dependiente del tipo de tratamiento elegido.

El propósito de este artículo es el de presentar un caso particular de un FD localizado en la región molar del maxilar en un paciente pediátrico de 4 años de edad. Hasta el momento, sólo se han reportado 13 casos de FD involucrados en la zona maxilar ${ }^{14}$, haciendo este caso aún más interesante.

\section{Reporte de Caso}

Se presenta un paciente masculino de 4 años de edad a consulta para ser valorado en conjunto por la Cátedra de Odontopediatría y la Pasantía de Patología y Cirugía Oral de la Universidad Latina de Costa Rica, por presentar una tumefacción generalizada a nivel de la encía tanto vestibular como palatina del cuadrante superior izquierdo, de alrededor de 2 años de evolución. No presenta antecedentes patológicos personales. Los padres autorizaron el tratamiento y la publicación del caso, de acuerdo con los parámetros utilizados en la Universidad Latina de Costa Rica.

Extrabucalmente hay muy poca evidencia de la alteración, todas las estructuras anatómicas se encuentran dentro de los límites normales (Figura 1), al examinar al paciente más 
detalladamente y desde superior (Figura 2) se determina un leve aumento de volumen el cual genera asimetría, correspondiendo con el área afectada.

Intrabucalmente se observa una tumefacción generalizada del cuadrante 2, abarcando específicamente desde distal de la pieza dental 6.3 hasta la tuberosidad maxilar (Figura 3). Tanto la encía adherida como la marginal presentan un color y apariencia normal. La zona afectada es dura y totalmente indolora a la palpación, no hay movilidad dental, hay ausencia clínica de procesos cariosos en las molares de la región, las pruebas de vitalidad pulpar realizadas en los dientes involucrados resultaron dentro de los límites normales. A parte de la tumefacción, no hay signos o síntomas de infección asociados.

Al comparar el cuadrante 1 con el cuadrante 2, las alteraciones se tornan más evidentes (Figura 4). Las piezas 6.4 y 6.5 tienen la apariencia de

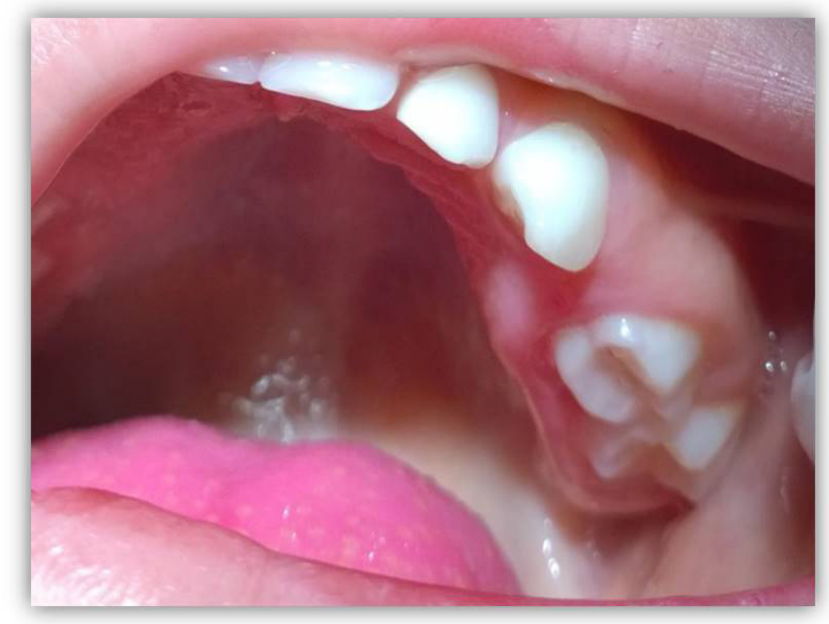

Figura 3. Aspecto clínico de lesión a nivel del cuadrante 2. Nótese el desplazamiento hacia distal de las piezas 6.4 y 6.5

estar ligeramente sumergidas dentro de la encía, de igual forma, estas piezas parecen encontrarse desplazadas hacia distal, generando un notable espacio o diastema entre ellas y la pieza dental 6.3. Lo más relevante del caso es la discrepancia entre el ancho palato-bucal del cuadrante 1 y el cuadrante 2.

Figura 4. Fotografía comparativa entre el cuadrante 1 y la tumefacción generalizada en cuadrante 2, la cual genera una notable asimetría

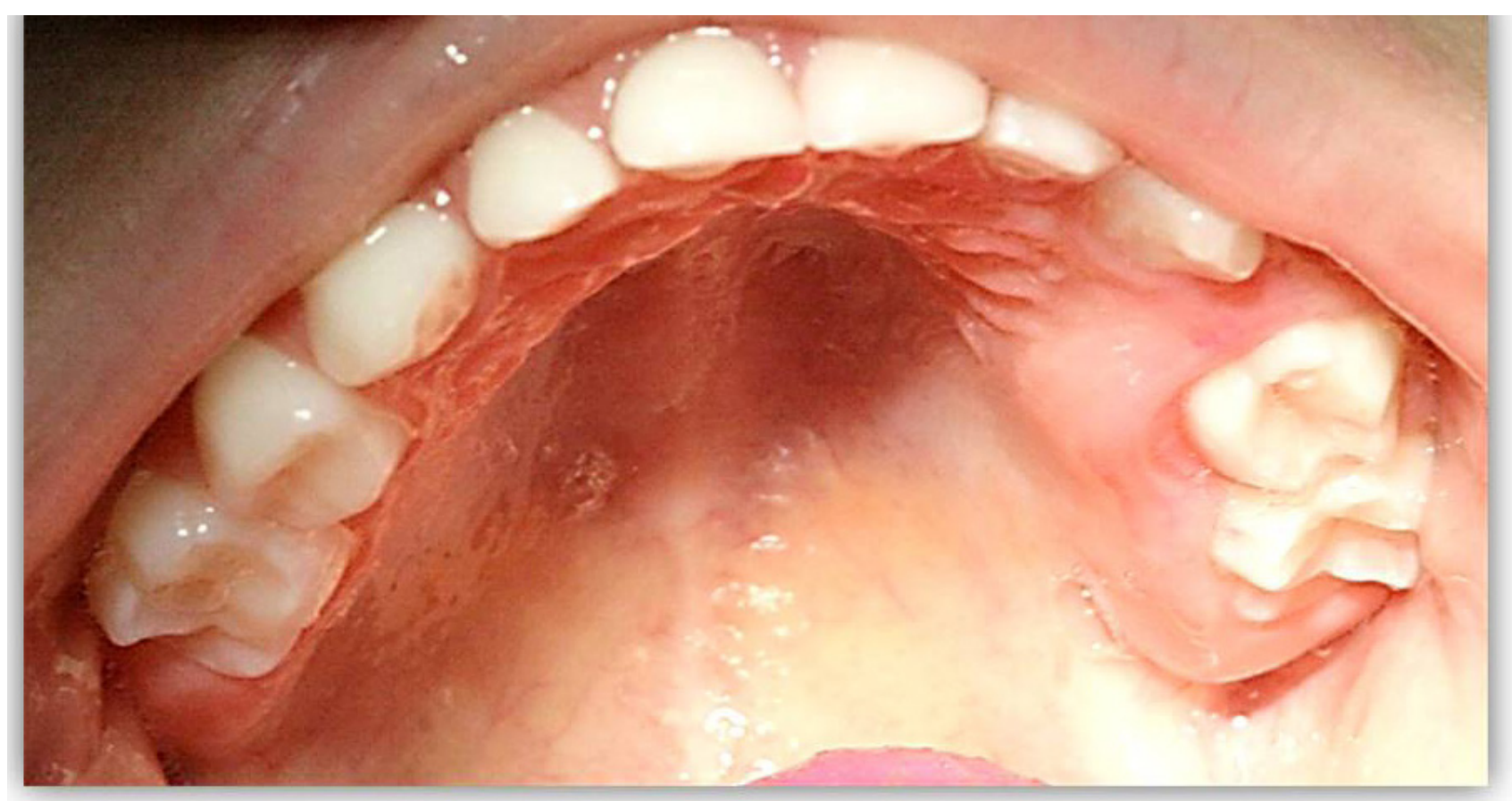




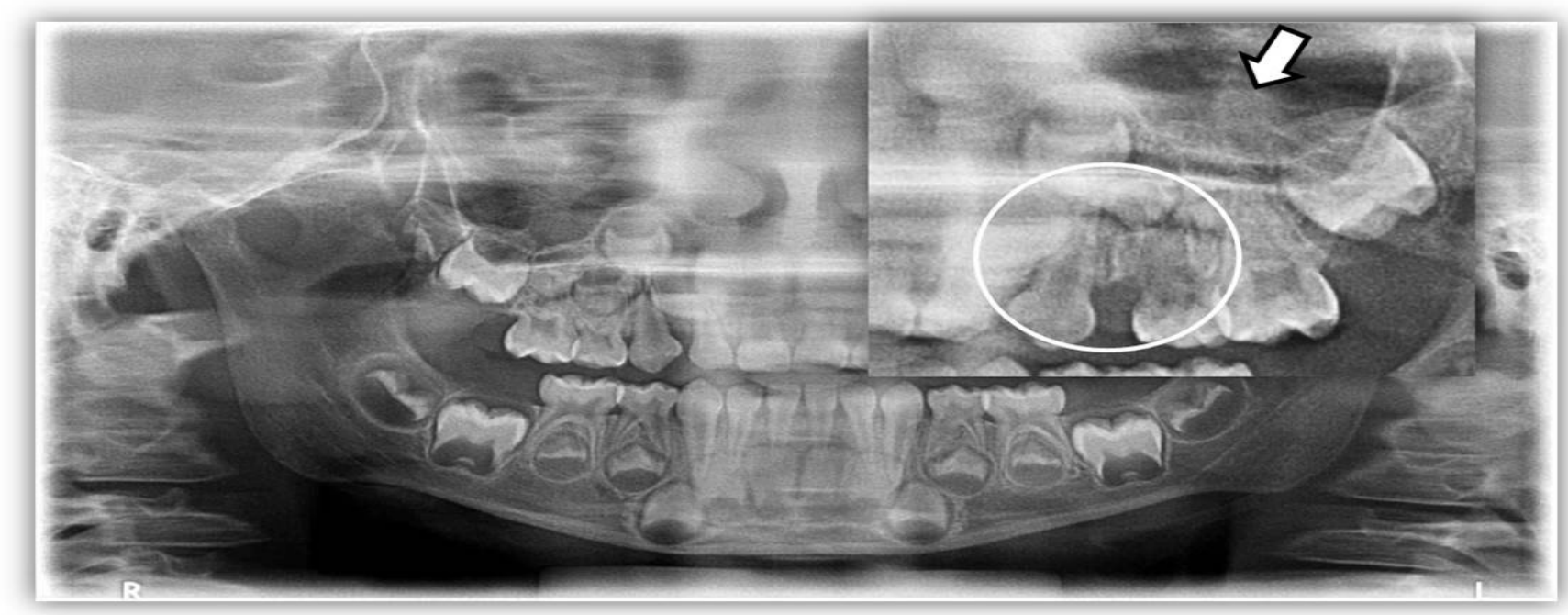

Figura 5.

Al revisar la ortopantomografía se observa un aparente proceso osteolítico difuso pero localizado, así como destrucción parcial de la pieza 6.4. A nivel del tercio inferior del seno maxilar izquierdo se detalla una lesión radiopaca, con una radiodensidad similar a la del hueso circundante, presentando un borde delimitable y continuo (Figura 5). Se toma una radiografía periapical de la región, la cual confirma un proceso osteolítico, con márgenes irregulares y presencia de espículas óseas. No hay evidencia de bordes escleróticos, el hueso circundante presenta una muy leve pérdida de su trabeculado. Hay evidencia de reabsorción de la raíz mesial de la pieza dental 6.4, de igual forma la furca se encuentra parcialmente reabsorbida con pérdida evidente de estructura dental, la raíz distal presenta ensanchamiento
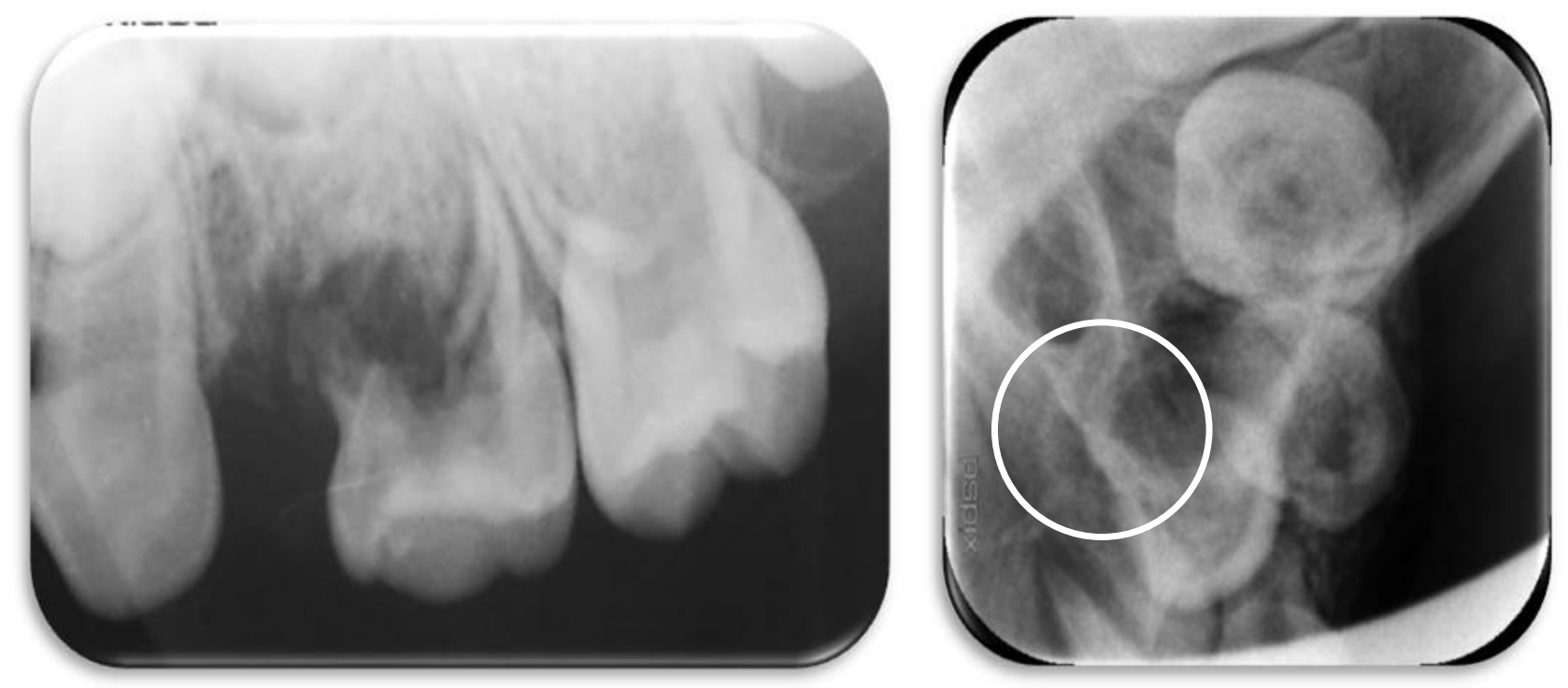

Figura 6 y 7. Radiografía periapical y oclusal mostrando proceso osteolítico de bordes pobremente definidos, con ausencia de reacción periostal 


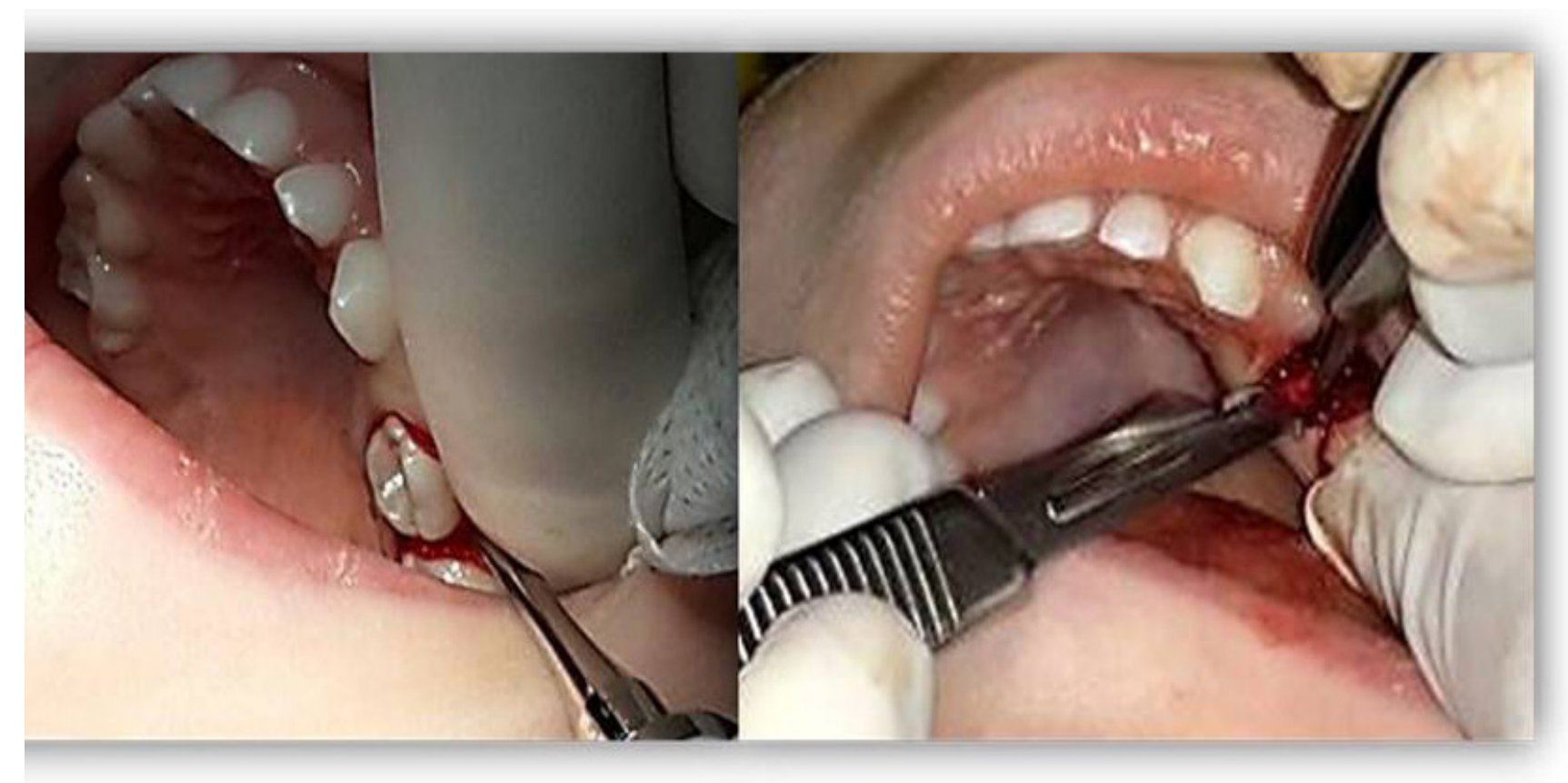

Figura 8, 9.

del espacio de la membrana periodontal (Figura 6). El estudio de la radiografía oclusal muestra ausencia de reacción periostal (Figura 7).

A razón de los hallazgos ya mencionados y en necesidad de otros medios diagnósticos que logren arrojar mayor información para avanzar el caso hacia la claridad, se indica la toma de una tomografía computarizada con cone beam, y se coordina una cita para realizar una intervención quirúrgica con el propósito de extraer la pieza dental 6.4 y tomar muestras de la zona para su respectivo estudio histopatológico. El diagnóstico clínico provisional otorgado para este caso fue el de un ameloblastoma periférico, dentro de los diagnósticos diferenciales se pensó en una osteomielitis, en una histiocitosis de células de Langerhans o en un linfoma no-Hodgkin.

\section{Intervención Quirúrgica}

Este procedimiento fue llevado a cabo mediante un abordaje multidisciplinario incluyendo el área de pediatría, cirugía y medicina bucal. Por razones de manejo físico, el paciente tuvo que ser inmovilizado y se tuvo que usar técnicas de manejo de la conducta como el control de la voz, decirmostrar hacer y refuerzo positivo entre otras, por lo que se requirió del odontopediatra para el manejo y realizar el procedimiento planificado. No se optó por utilizar sedación ya que no es un procedimiento realizado dentro de la institución. La intervención se realizó bajo anestesia local, utilizando un cartucho conteniendo lidocaína al $2 \%$ con epinefrina 1:100.000. Se prosigue a luxar la pieza dental afectada (Figura 8), percibiéndose la debilidad estructural del diente al generarse una sensación de "arenilla" mientras se efectúan los movimientos exodónticos. Se logra avulsionar la pieza dental, seguidamente se dificulta la toma de las muestras, ya que tanto el tejido duro como el blando se encontraban sumamente lábiles y se desgarraban con facilidad. Finalmente se colocan 2 muestras importantes de tejido blando y duro (Figura 9), junto con la corona de la pieza 6.4 en un frasco con formalina al $10 \%$ para su posterior análisis histopatológico. 


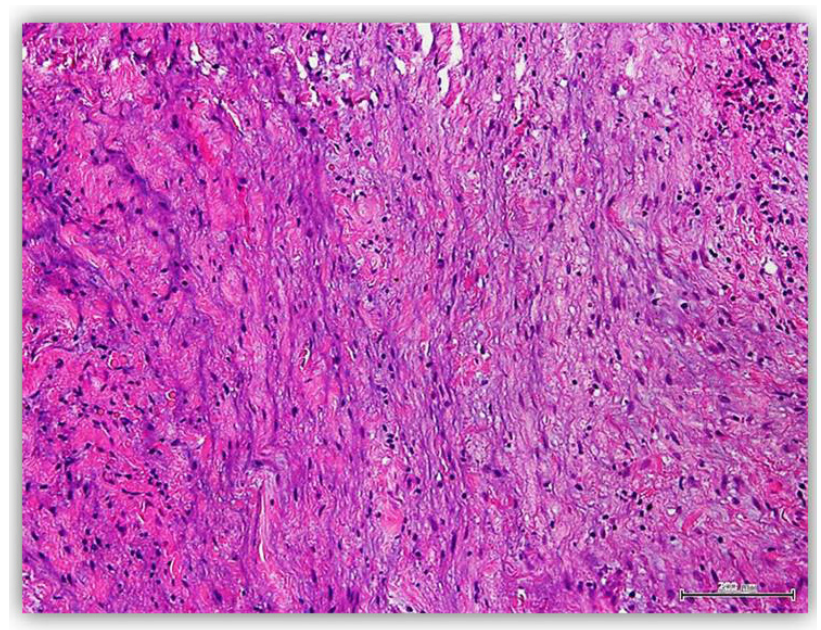

Figura 10.

Se coloca un punto en colchonero utilizando sutura de seda 3-0. Cabe destacar que durante la intervención quirúrgica, no hubo signos de exudado purulento ni mal olor, descartando definitivamente un proceso infecto-inflamatorio de trasfondo.

\section{Resultado Histopatológico}

A nivel microscópico las muestras develan zonas con fibroblastos pequeños y alargados, otras zonas presentan fibroblastos más gruesos y circunferenciales, ambos dentro de un abundante estroma de fibras colágenas, algunas dispuestas en bandas paralelas y condensadas entre sí (Figura 10). El grado de celularidad varía dependiendo de la zona estudiada. No hay presencia de atipia celular, se observan pocos signos de mitosis, hay ausencia de infiltrado linfocítico importante. En otras zonas de la muestra se observan microespículas óseas, probablemente siendo restos del trabeculadoóseo que no fue reabsorbido en su totalidad (Figura 11), es oportuno mencionar que el parénquima tumoral no produce neoformación ósea. En el

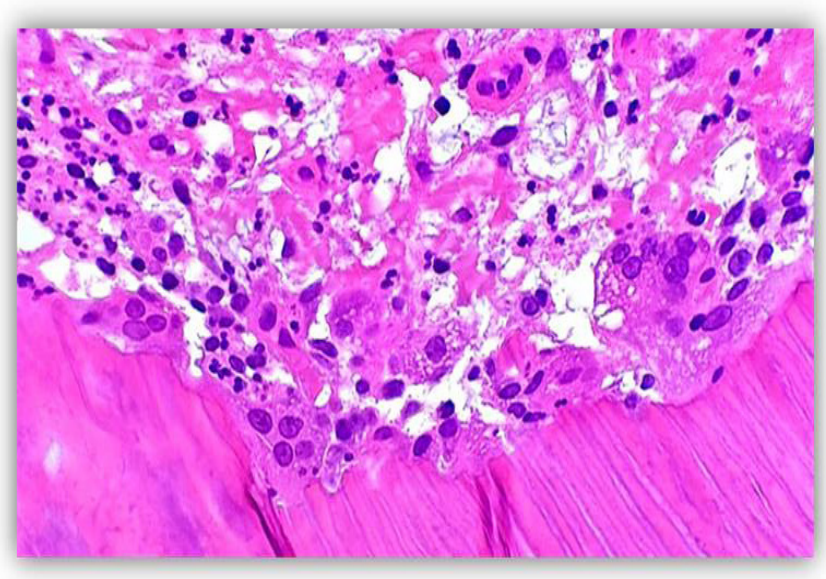

Figura 11.

corte histológico del tejido dental se observan múltiples osteoclastos íntimamente relacionados con una superficie dentinal altamente irregular (Figura 12).

Con la suma de los hallazgos clínicos, radiológicos e histopatológicos se genera el diagnóstico definitivo de un Fibroma Desmoplásico. El caso fue referido a las autoridades competentes del Hospital Nacional de Niños de Costa Rica para su continuidad y respectivo tratamiento. Por su crecimiento aparentemente lento y por la ausencia de perforaciones en las tablas corticales e invasión a tejido blando, el tratamiento y manejo

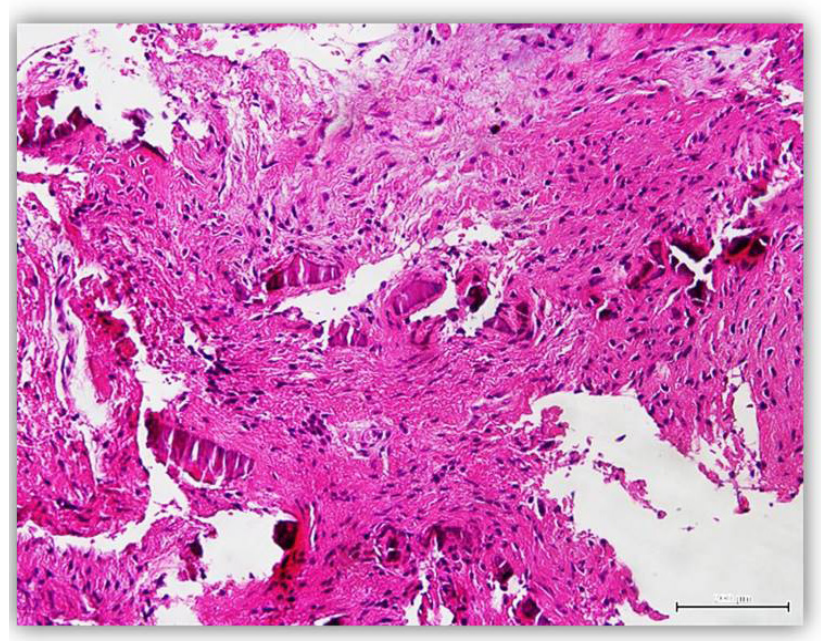

Figura 12. 
recomendado para este caso en particular es el del curetaje. Actualmente el paciente no ha sido intervenido quirúrgicamente por las autoridades correspondientes, ya que han decidido mantener la lesión en observación.

\section{Discusión}

El FD es una entidad de etiología desconocida que puede desarrollarse en cualquier hueso del cuerpo humano ${ }^{7}$. Aunque se concuerda que la mandíbula representa el cuarto lugar de desarrollo del $\mathrm{FD}^{4}$, distintos estudios difieren afirmando que la mandíbula es el hueso más comúnmente afectado, seguido por el fémur, la pelvis, el húmero y radio, todos en frecuencia decreciente de aparición ${ }^{3,8,13}$. A pesar de su alta agresividad local, el FD no produce metástasis, y aunque generalmente se desarrolla de forma solitaria $^{15}$, se ha descrito el caso de un FD que se presentaba en forma multifocal a ambos lados de la mandíbula 6 .

Entre las descripciones clínicas realizadas por parte de otros reportes encontramos desde una tumefacción leve y generalizada a nivel del hueso alveolar, lesiones de apariencia exofítica localizadas, hasta agrandamientos que involucran tejido blando simulando en apariencia una celulitis facial. La gran mayoría de casos se presentan asintomáticos, siendo el principal signo el agrandamiento de la zona afectada. Otros signos documentados refieren movilidad dental, desplazamiento dental, cambios en la oclusión, pérdida de la función y fracturas patológicas ${ }^{2,8}$. Se reporta dolor localizado en la región de afectación en un $15 \%$ de los $\operatorname{casos}^{3}$. La encía que recubre al hueso alterado se presenta con un color y textura normal a menos que se halle ulcerada o traumatizada secundario al agrandamiento patológico. Puede haber infección de la lesión². Por su crecimiento lento e insidioso y por ser de comienzo asintomático, el FD por lo general se diagnostica de manera tardía?.

Los hallazgos radiográficos tampoco son sugerentes $^{13}$, esto a razón de su variabilidad en comportamiento y aspecto. Algunas lesiones se muestran multiloculares, otras uniloculares ${ }^{9}$, ambas pueden presentarse con márgenes bien definidos en ausencia de un patrón esclerótico, o como lesiones osteolíticas pobremente delimitadas e irregulares, con o sin presencia de espículas óseas ${ }^{7,3,10}$. Otros autores describen márgenes escleróticos rodeando la lesión y/o pérdida del trabeculado óseo ${ }^{3,9}$. Lo que sí comparten todos los reportes es la tendencia de expandir, erosionar y hasta perforar la tabla cortical de la zona afectada, invadiendo el tejido blando vecino. No produce alteración periostal $^{4,10}$.

Los hallazgos histopatológicos son los más estables en cuanto a guiar el caso hacia un diagnóstico definitivo. A este nivel, el FD se destaca por ser una lesión no encapsulada, lo que dificulta la identificación microscópica del margen de la misma ${ }^{12}$. Se desarrolla reabsorbiendo e infiltrando el hueso trabecular ${ }^{17}$, con aposición de grandes cantidades de tejido fibroso por parte de una proliferación fibroblástica. Estos fibroblastos se presentan pequeños y alargados dentro de abundantes fibras colágenas dispuestas de forma paralela generando la apariencia de bandas. El grado de celularidad puede variar de área en área dentro de una misma lesión, en las zonas más celulares los fibroblastos se observan más gruesos y redondos dentro de un estroma con menos colágeno ${ }^{10}$. No hay 
atipia celular, hay pocos eventos mitóticos ${ }^{3,1}$. Puede haber ausencia o presencia de un leve infiltrado inflamatorio. En algunos casos se reportan microespículas óseas dentro de la lesión ${ }^{10}$, las cuales probablemente son restos del trabeculado óseo que falló en ser reabsorbido.

Usualmente se puede llegar al diagnóstico de un FD a través de la suma de los hallazgos clínicos, radiológicos e histopatológicos, aun así, existen otros métodos coadyuvantes en el proceso diagnóstico como lo es la inmunohistoquímica, y aunque no hay un marcador específico para diagnosticar al FD, algunos especialistas refieren haber tenido éxito al utilizar los marcadores de actina de músculo liso, vimentina, $\beta$-catenina $y$ factor XIIIa 3, 4, 9, 13, 18, por ser positivo a la actina de músculo liso ciertos autores lo consideran un tumor miofibroblástico. Se ha confirmado útil el uso del marcador fluorodesoxiglucosa en la tomografía por emisión de positrones, especialmente en la detección de lesiones incipientes o inclusive en el desarrollo de una lesión recidiva posterior a la intervención .quirúrgica ${ }^{17}$

El tratamiento debe ser adaptado a cada caso, tomando en cuenta las características de agresividad y destrucción local que pueda presentar la lesión ${ }^{10}$. En general, el tratamiento por excelencia es la resección radical o escisión quirúrgica amplia, ya que la recurrencia es sumamente alta al optar por otros métodos como lo es el curetaje, por medio del cual se reporta un $70 \%$ de recidiva ${ }^{3,10}$. No se recomienda el uso de radioterapia, ya que este procedimiento implica la posibilidad de generar un efecto mutagénico sobre las células de la lesión, trasformando una patología estrictamente benigna en una con potencial metastásico. Otros autores refieren una relación entre lesiones con alta celularidad a una mayor tendencia a recurrir ${ }^{5}$. La reconstrucción anatómica post-cirugía se puede lograr a través de injertos autólogos, ya sean obtenidos de la cresta ilíaca o de las costillas, claro está en los .casos que lo ameriten ${ }^{11}$

\section{Conclusiones}

Se reporta el caso de un paciente pediátrico presentando un FD en maxilar. Radiográficamente nuestro caso asemejaba un proceso infeccioso compatible con una osteomielitis, aunque esto no fue congruente con la clínica encontrada en el paciente, por ello la sospecha y la necesidad de realizar la toma de una muestra para su estudio histopatológico. Este caso fue diagnosticado satisfactoriamente a una edad muy temprana, en donde la lesión no presentaba un tamaño considerable. El paciente fue referido al Hospital Nacional de Niños en donde fue intervenido quirúrgicamente y se .encuentra bajo observación

\section{Referencias bibliográficas}

1. Herford, A. Reder, P. Ducic, Y. Multifocal Desmoplastic Fibromas of the Mandible JOMS. 2001: 59: $1078-1081$.

2. Acosta, M. Infante, C. López, D. Carranza, A. Gutiérrez, J. Fibroma desmoplásico de mandíbula asociado a esclerosis tuberosa. Revisión de literatura y presentación de un caso. Rev Esp de Cir Oral y Maxilofac. 2008: 30: 107-114.

3. Basu, S. Abhishek, S. Swetha, S. Desmoplastic fibroma of mandible and skull: A case report and review of literature. Indian Journal of Stomatology. 2010: 1:96-99. 
4. Dhaif, G. Satir, A. Sharif, S. Desmoplastic Fibroma of the Mandible: A 5 Year Follow-Up. Bahrain Medical Bulletin. 2008: 304: $1-10$.

5. Guru, K. Gupta, M. Pillai, A. Moghe, S. Satpathy M. Desmoplastic Fibroma of the Maxilla: Report of a Rare Case. People's J. Scient. Res. 2012: 6: 36-38.

6. Jamali, M. Desmoplastic Fibroma Report of a Rare Lesion in Unusual Craniofacial Location. NYSDJ. 2013: 43-45.

7. Min Kang, D. Juhng, S. Sohn, Y. Soo Kim, H. Imaging findings of desmoplastic fibroma rarely involving the clavicle: case report. KJR. 2014: 15:130-133.

8. Licéaga, C. Mosqueda, A. Velázquez, G. Ortiz, E. Fibroma Desmoplásico, reporte de un caso y revisión de literatura.

Rev Esp de Cir Oral y Maxilofac. 2009: 32:21-24.

9. Lucchesi, Francisco. Egito, B. Bessa-nogueira, R. Desmoplastic Fibroma of the mandible in a child: case report. IJOS. 2007: 69:254-256.

10. Neville, B. Damm, D. Allen, C. Bouquot, J. Oral and Maxillofacial Pathology. Saunders Company. 2002: 573-74.

11. Perotta, F. Fretes, G. Navarro, A. Schaerer, C. Cubilla, A. Fibroma desmoplásico mandibular. Mem.Inst.Invest. Cienc.Salud. 2006: 4: 43-45.

12. Reid, E. Lawoyin, D. Suresh, L. Longwe, E. Desmoplastic fibromas of the anterior mandible case report and review of literature. NYSDJ. 2009: 33-34.

13. Schneider, M. Zimmermann, A. Depprich, R. Kübler, N. Engers, R. Naujoks, C. Handschel, J. Desmoplastic fibroma of the mandible - review of literature and presentation of a rare case. Head-Face-Med. 2009: 5:25.

14. Tandon, S. Kumar, R. Inrtaoral desmoplastic fibromas: a manifestation of tuberous sclerosis. Fetal Pediat Pathol. 2012: 31:195-201.

15. Shi, H. Wang, P. Wang, S. Yu, Q. Case report: Desmoplastic fibroma of the mandible. Dentomaxillofacial Radiology. 2008: $37,408-411$.

16. Barnes, L. Surgical Pathology of Head and Neck. Informa Healthcare. 2009: 987-988, 1279.

17. Taketo, O. Saito, T. Takagi, T. Suehara, Y. Desmoplastic fibroma of the rib with cystic change: a case report and literature review. Skeletal Radiology. 2013: 6:2230-2236.

Recibido 20/05/2015

Aceptado 10/09/2015

Correspondencia: Joseph Ulate Jiménez julatej@gmail.com Facultad de Odontología,

Universidad Latina de Costa Rica, sede San Pedro, San José Costa Rica.

Teléfono: (506) 2234-0785, (506) 8332-5702 